\title{
Luminescence from erbium-doped silicon nanocrystals in silica: Excitation mechanisms
}

\author{
A. J. Kenyon, ${ }^{\text {a) }}$ C. E. Chryssou, and C. W. Pitt \\ Department of Electronic and Electrical Engineering, University College London, Torrington Place, \\ London WC1E 7JE, United Kingdom \\ T. Shimizu-Iwayama \\ Department of Materials Science, Aichi University of Education, Igaya-cho, Kariya-shi, \\ Aichi 448-8542, Japan \\ D. E. Hole \\ School of Engineering, University of Sussex, Falmer, Brighton BN1 9QH, United Kingdom \\ N. Sharma and C. J. Humphreys \\ Department of Materials Science and Metallurgy, University of Cambridge, Pembroke Street, \\ Cambridge CB2 3QZ, United Kingdom
}

(Received 13 February 2001; accepted for publication 24 September 2001)

\begin{abstract}
We develop a model for the excitation of erbium ions in erbium-doped silicon nanocrystals via coupling from confined excitons generated within the silicon nanoclusters. The model provides a phenomenological picture of the exchange mechanism and allows us to evaluate an effective absorption cross section for erbium of up to $7.3 \times 10^{-17} \mathrm{~cm}^{2}$ : four orders of magnitude higher than in stoichiometric silica. We address the origin of the $1.6 \mathrm{eV}$ emission band associated with the silicon nanoclusters and determine absorption cross sections and excitonic lifetimes for nanoclusters in silica which are of the order of $1.02 \times 10^{-16} \mathrm{~cm}^{2}$ and $20-100 \mu \mathrm{s}$, respectively. (C) 2002 American Institute of Physics. [DOI: 10.1063/1.1419210]
\end{abstract}

\section{INTRODUCTION}

Light emission from rare-earth doped materials is a rapidly growing field thanks to the promise of integration of existing semiconductor technology with emission at technologically important wavelengths (primarily $1535 \mathrm{~nm}$ for telecommunications, but also red, blue, and green for displays applications). ${ }^{1}$ A major driving force behind this development is the requirement for a wide range of cheap and compact optical components for implementation of wavelength division multiplexing (WDM) in fiber-to-the-home systems. The increasing demand for the Internet and other broadband telecommunications services is driving an expansion of optoelectronics technologies in this area, and there is currently a move towards integrating many of the required optical components onto silicon chips. A key requirement is therefore for a silicon/silica-based gain element operating at $1.5 \mu \mathrm{m}$ that can readily be integrated with existing semiconductor and fiber technologies. $\mathrm{Er}^{3+}$ is particularly attractive because its emission wavelengths, around $1.53 \mu \mathrm{m}$, coincide with the low attenuation region of silica optical fibers.

During recent years, strong, room temperature, visible light emission from novel forms of silicon, including porous silicon, ${ }^{2}$ and silicon nanoclusters, ${ }^{3-7}$ has been demonstrated. Luminescence from this material characteristically exhibits two distinct bands centred around $1.6 \mathrm{eV}$ and $2-2.2 \mathrm{eV}$. The higher energy band is generally thought to be due to the presence of oxygen vacancy-related defects (generically similar to the nonbridging oxygen hole center, $\mathrm{NBOHC}$ ), $, 8,9$

\footnotetext{
a)Electronic mail: t.kenyon@ee.ucl.ac.uk
}

while that at $\sim 1.6 \mathrm{eV}$ is variously ascribed to the radiative recombination of confined excitons within the silicon nanoclusters, ${ }^{4}$ "luminescent centers," 10 or interfacial states. ${ }^{11,12}$ There remains controversy over the species responsible for this emission, ${ }^{13}$ but despite this uncertainty, a number of prototype devices have been manufactured from porous silicon. ${ }^{14}$ There have also been demonstrations of electroluminescence from nanoclustered silicon, ${ }^{15-17}$ opening up the possibility of light emission from silicon based materials. However, neither of these classes of material provide emission in the technologically important $1.5 \mu \mathrm{m}$ band needed for telecommunications applications. Recently, $\mathrm{Er}^{3+}$ doped porous or nanoclustered silicon has been attracting strong interest thanks to the possibility of incorporating $1.53 \mu \mathrm{m}$ erbium emission with mature semiconductor processing technologies. ${ }^{1,78-22}$ Recent studies have demonstrated that $\mathrm{Er}^{3+}$ doped silica containing silicon nanoclusters produced by cosputtering, ${ }^{23}$ plasma-enhanced chemical vapor deposition (PECVD), ${ }^{1821,24}$ or ion implantation ${ }^{25-27}$ exhibits a strong coupling between $n c-\mathrm{Si}$ and $\mathrm{Er}^{3+}$ that results in excitation being transferred from the broad-band absorbing silicon nanoclusters to the narrow-band emission from the rare-earth ion. Indeed, it has been demonstrated that the photoluminescence (PL) yield of $\mathrm{Er}^{3+}$ in so-called "siliconrich silica" is at least an order of magnitude larger than that from $\mathrm{Er}^{3+}$ in stoichiometric silica. ${ }^{25}$ In addition, it has been shown that, due to interaction between silicon nanoclusters and $\mathrm{Er}^{3+}$, it is possible to excite $\mathrm{Er}^{3+}$ ions indirectly using pump wavelengths that do not correspond to any of the 
(a)

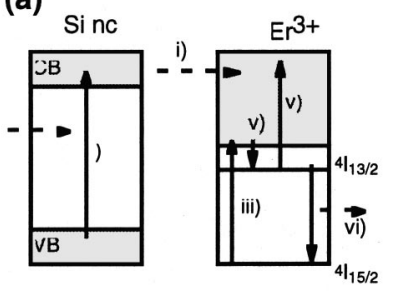

(b)

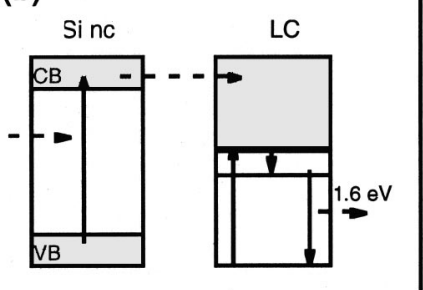

(c)

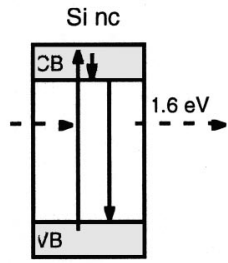

FIG. 1. Schematic mechanisms for (a) excitation exchange between silicon nanoclusters and erbium ions, (b) luminescence from luminescent centers associated with nanoclusters, (c) luminescence from radiative recombination of confined excitons. The upper states of the rare-earth ion and luminescent center are shaded to indicate that we have no information about which levels are accessed. The numbered arrows in (a) refer to: (i) creation of an electron-hole pair by absorption of a photon, (ii) excitation exchange, (iii) promotion of an electron from the ground state of erbium to an unspecified excited state, (iv) decay to the metastable state, (v) upconversion, (vi) emission of a $1535 \mathrm{~nm}$ photon.

principal $\mathrm{Er}^{3+}$ absorption bands. ${ }^{28}$ This coupling mechanism is very important since it can relax the requirements on the $\mathrm{Er}^{3+}$ pump wavelength leading to the production of broadband pumpable optical amplifiers at the important telecommunications wavelengths around $1.55 \mu \mathrm{m}$.

This excitation exchange mechanism between silicon nanoclusters and $\mathrm{Er}^{3+}$ has recently been studied theoretically using two approaches. ${ }^{10,29}$ That due to Qin et al. ${ }^{29}$ is based on the quantum-confinement luminescent center (QCLC) model and suggests that the excitation of $\mathrm{Er}^{3+}$ ions takes place via tunneling of optically generated free electrons from the $n c-\mathrm{Si}$ to $\mathrm{Er}^{3+}$. The second study, by Franzò et al., ${ }^{10} \mathrm{de}-$ scribes the dynamics of the $n c-\mathrm{Si} / \mathrm{Er}^{3+}$ system using a rate equation approach. Figure 1(a) illustrates a generic exchange mechanism consisting of an initial optical absorption step producing an exciton within the silicon nanocluster, an exchange, and consequent excitation and deexcitation of the rare-earth ions. Also included is an upconversion step in which a rare-earth ion already in the ${ }^{4} I_{13 / 2}$ metastable state is promoted to higher states by further interaction with an excited nanocluster. We do not know which of the higher states of the $\mathrm{Er}^{3+}$ ion are populated by transfer from the silicon nanocluster, and as a consequence we treat both the cluster and excited rare-earth ion as quasi-two-level systems. The nature of the exchange is not defined in Fig. 1(a), and in this article we assume resonant interactions between $\mathrm{Er}^{3+}$ and nanoclusters along the lines of the Förster-Dexter energy transfer model. We consider separately the 1.6 and $0.85 \mathrm{eV}$ $(1535 \mathrm{~nm})$ emission bands. The latter is characteristic of the ${ }^{4} I_{13 / 2}-{ }^{4} I_{15 / 2} \mathrm{Er}^{3+}$ transition, while the origin of the former, although associated with silicon nanoclusters, remains a subject for debate. ${ }^{13}$ Figures 1 (b) and 1(c) illustrate two possible

schemes for the $1.6 \mathrm{eV}$ emission: radiative recombination of confined excitons [Fig. 1(b)] and transfer to a "luminescent center" [Fig. 1(c)]. In this study, we identify a series of experiments that may be performed to distinguish between the two models of the $1.6 \mathrm{eV}$ band, and we evaluate an effective absorption cross section for erbium in silicon-rich silica. For the samples produced in this study, this is up to four orders of magnitude larger than the direct optical absorption cross section for $\mathrm{Er}^{3+}$ in stoichiometric silica.

\section{EXPERIMENT}

Thin film samples were prepared by two methods; the first being PECVD onto silicon wafers. This method is more fully detailed elsewhere, ${ }^{5,18}$ but briefly consisted of plasma dissociation of silane, nitrous oxide, and a volatile erbium organic chelate in a parallel-plate plasma chamber. Film stoichiometry was controlled by varying the relative flow rates of the reagent gases, and by controlling both the temperature of the organic precursor and the flow rate of the carrier gas. Auger analysis of the deposited films showed them to be silicon-rich (7\% excess silicon) and to contain 1 at. \% erbium. Film thicknesses were in the range $1-3 \mu \mathrm{m}$. Previous work has demonstrated that careful selection of growth conditions produces films containing excess silicon in the form of nanoclusters, the size of which depends on growth parameters and post-process annealing. ${ }^{5,30}$ In this study, samples were annealed at 900 and $1100{ }^{\circ} \mathrm{C}$ for $90 \mathrm{~min}$ in flowing argon. The presence of silicon clusters has been inferred from high resolution transmission electron microscopy (HRTEM), optical absorption, and visible photoluminescence studies of silicon-rich silica. 5,30,31

The second preparation method consisted of sequential ion implantation of high-quality thermally grown $\mathrm{SiO}_{2}$ layers on silicon substrates with $\mathrm{Si}^{+}$and $\mathrm{Er}^{3+}$ ions. Multiple implants of both silicon and erbium were used in order to obtain flat concentration profiles with depth. Again, this method is more thoroughly detailed elsewhere. ${ }^{25,30}$ In all cases, samples were annealed at $1050^{\circ} \mathrm{C}$ in nitrogen for $8 \mathrm{~h}$ following the silicon implant: these annealing conditions are known to produce silicon nanocrystals with a diameter around $3 \mathrm{~nm}$. Care was taken to ensure that the subsequent erbium implants (peak concentration 0.5 at. \% in each case) overlapped strongly with the silicon-rich region.

Room temperature photoluminescence experiments were conducted using an argon-ion laser, a scanning monochromator, a photomultiplier tube for $1.6 \mathrm{eV}$ emission, and an InGaAs photodiode for detection of erbium luminescence. Standard lock-in techniques were employed for detection. In addition, measurements of luminescence dynamics were made by modulating the laser excitation using a rotating chopper blade and displaying the luminescence signal on a digital sampling oscilloscope (LeCroy Model 9310). Chopping frequencies were in the range $200-500 \mathrm{~Hz}$, and the time resolution of our measurement system (limited by the chopper speed and amplifier bandwidth) was of the order of $15 \mu \mathrm{s}$. From these measurements, luminescence rise times, and radiative lifetimes were extracted by fitting the oscilloscope traces with exponential functions. Measurements were 
also made of the dependence of luminescence output at 1.6 $\mathrm{eV}$ and $1535 \mathrm{~nm}$ on input pump power.

\section{MODEL}

Our results, and those from other studies on similar materials, ${ }^{10,18,21,23,24,25}$ clearly demonstrate a strong coupling mechanism between the silicon nanocrystals and erbium ions. The absorption process is dominated by the silicon nanocrystals, which transfer excitation to the optically active rare-earth ions. The image is complicated by the presence of two distinct emission bands: that associated with silicon nanoclusters $(1.6 \mathrm{eV})$, and the characteristic intra- $4 f \mathrm{Er}^{3+}$ transition $(0.8 \mathrm{eV}$ or $1535 \mathrm{~nm})$. Photoluminescence results indicate that the two luminescence mechanisms are in competition: the presence of optically active erbium ions quenches the $1.6 \mathrm{eV}$ emission, and there is an inverse relationship between erbium concentration and $1.6 \mathrm{eV}$ emission. ${ }^{10,28}$ An important point to note is that although the presence of erbium quenches the $1.6 \mathrm{eV}$ emission band, as widely demonstrated in the literature, ${ }^{10,27}$ emission in this region was detected for all samples studied. The intensity of the $1.6 \mathrm{eV}$ emission decreased sharply when erbium was implanted into the silicon-rich samples, but was still detectable. Even for erbium concentrations as high as 1 at. \%, $1.6 \mathrm{eV}$ emission was observed in the PECVD samples. Although it is possible that this is from an erbium implant tail in which nanocrystals are present while Er is almost absent, this cannot be the case for the PECVD grown material in which both nanocrystals and erbium are uniformly distributed throughout the film. We therefore conclude that the 1.6 $\mathrm{eV}$ emission is not completely quenched even at erbium concentrations up to 1 at. \%. We take a phenomenological approach to modeling the excitation exchange process in which we consider each luminescence band separately.

\section{A. $1.6 \mathrm{eV}$ emission}

Any discussion of the exchange mechanism must include a consideration of the nature and role of the species responsible for the $1.6 \mathrm{eV}$ emission band. It is an intrinsic property of the matrix, and it is evident that this emission process is in competition with that from the rare-earth ion (though this observation does not in itself say anything about the exchange mechanism). There is good agreement that the optical absorption process in nanoclustered silicon is governed by quantum confinement effects, and there have been a number of reports of a dependence of absorption band edge on nanocluster size. ${ }^{32}$ However, there remains controversy over the role of quantum confinement in subsequent emission: observation in some cases of a $1.6 \mathrm{eV}$ luminescence peak whose energy is independent of nanocluster size has led to the postulation of an excitation exchange mechanism from optically generated excitons to a luminescent center, possibly related to surface states at the nanocluster/silica matrix boundary. ${ }^{10-12}$ Results in the literature are far from consistent on this point. Some groups report no shift in luminescence peak energy with annealing, ${ }^{11}$ while other work shows a clear redshift of the luminescence band on annealing. ${ }^{5}$ High temperature annealing is associated with "Ostwald ripening" of silicon nanoclusters, leading to cluster growth: the redshift in luminescence is therefore correlated with increased cluster size in the quantum confinement model. Absence of this redshift is generally taken as evidence against the excitonic recombination model. This aside, the excitonic recombination model is a single-step process, whereas the luminescent center model involves absorption by one species (excitons) followed by an exchange step to the luminescent species. This implies that a study of the dynamics of the $1.6 \mathrm{eV}$ emission band may yield clues to its origin.

To investigate this further, we have set up rate equations governing the populations of excitons and luminescent centers for the two models (direct excitonic recombination, or luminescent centers). First, we consider the case of radiative recombination of confined excitons [Fig. 1(b)]. The following equation governs exciton generation and destruction:

$$
\frac{d N_{\mathrm{exc}}}{d t}=\sigma \phi\left(k N-N_{\mathrm{exc}}\right)-\frac{N_{\mathrm{exc}}}{\tau} .
$$

Here, the first term shows that the number of excitons $\left(N_{\text {exc }}\right)$ is proportional to the pump photon flux $(\phi)$, the absorption cross section $(\sigma)$, the concentration of nanocrystals $(N)$, and is limited by a factor $k$ that governs the maximum number of excitons that can exist on a single cluster. This last factor is not included in Franzò's treatment, ${ }^{10}$ but is a necessary limiting factor to prevent the number of excitons exceeding, ultimately, the total number of excess silicon atoms. The second term is the recombination term responsible for luminescence. Note here that the lifetime $(\tau)$ is an effective lifetime that takes into account both radiative and nonradiative recombination. Thus,

$$
\frac{1}{\tau}=\frac{1}{\tau_{\text {rad }}}+\frac{1}{\tau_{\text {nonrad }}} .
$$

In this scenario, solution of the rate equation yields the time evolution of exciton luminescence, along with its dependence on pump photon flux. Assuming a rapidly rising incident excitation pulse and solving for the initial rise in exciton population, the solution is

$$
N_{\text {exc }}=\frac{\sigma \tau \phi k N}{\sigma \tau \phi+1}\left\{1-\exp \left[-\left(\sigma \phi+\frac{1}{\tau}\right) t\right]\right\} .
$$

The exponential term yields a characteristic initial rise time of exciton population (and hence, by inference, $1.6 \mathrm{eV}$ emission) of

$$
\frac{1}{\tau_{\text {rise }}}=\sigma \phi+\frac{1}{\tau} .
$$

Note that this is linearly dependent on photon flux $(\phi)$. Assuming recombination to be radiative at $1.6 \mathrm{eV}$, a plot of reciprocal luminescence rise time as a function of $\phi$ should therefore be a straight line, with $\sigma$ as its gradient, and $\tau$ its intercept.

Alternatively, considering the luminescent center model, we now assume the $1.6 \mathrm{eV}$ emission arises from a two-step process; generation of excitons within silicon nanoclusters followed by transfer to luminescent centers [Fig. 1(c)]. Now 
the rate equation governing the population of luminescent centers must be solved, in addition to that relating to the population of excitons. Here we write

$$
\frac{d N_{c}^{*}}{d t}=L^{*}-\frac{N_{c}^{*}}{\tau_{d}} .
$$

In this case, the first term determines the filling of the excited state of the luminescent center by transfer from excitons within the nanocluster, and the second term governs the 1.6 $\mathrm{eV}$ emission. $\tau_{\text {tr }}$ is a characteristic excitation transfer time, and $\tau_{d}$ the excited state lifetime. We write $L^{*}$ as

$$
L^{*}=\frac{N_{\mathrm{exc}} \eta_{c}\left(N_{c}-N_{c}^{*}\right) \Lambda}{\tau_{\mathrm{tr}}^{c}} .
$$

This is clearly contingent on the presence of an initial population of excitons. Here, $\eta_{c}$ is the quantum efficiency of filling of the excited state of the luminescent center, $\Lambda$ is an interaction volume which is related to the Förster radius, $R_{0}$ is in Förster-Dexter theory and defines the volume within which excitation exchange occurs, $\tau_{\text {tr }}^{c}$ is the characteristic excitation transfer time, and $N_{c}$ and $N_{c}^{*}$ are the populations of the ground state and excited states of the luminescent center, respectively. Equation (5) now becomes

$$
\frac{d N_{c}^{*}}{d t}=\frac{N_{\mathrm{exc}} \eta_{c}\left(N_{c}-N_{c}^{*}\right) \Lambda}{\tau_{\mathrm{tr}}^{c}}-\frac{N_{c}^{*}}{\tau_{d}^{c}} .
$$

Applying boundary conditions, and assuming that the population of excitons reaches a steady state well before that of the luminescent centers, the solution is

$$
\begin{aligned}
N_{c}^{*}= & \frac{N_{c}}{1+\frac{\tau_{\mathrm{tr}}^{c}}{\tau_{d}^{c} N \eta_{c} \Lambda}\left(\frac{\sigma \tau \phi+1}{\sigma \tau \phi k}\right)} \\
& \times\left\{1-\exp \left[-\left(\frac{N \eta_{c} \Lambda}{\tau_{\mathrm{tr}}^{c}} \frac{\sigma \tau \phi k}{\sigma \tau \phi+1}\right)+\frac{1}{\tau_{d}^{c}}\right] t\right\} .
\end{aligned}
$$

Note that in this case the time evolution of the luminescent center population is not linear with photon flux. A plot of reciprocal luminescence rise time against $\phi$ will now be a curve of the general form:

$$
\frac{1}{\tau_{\text {rise }}}=\left[\left(\frac{N \eta_{c} \Lambda}{\tau_{\mathrm{tr}}^{c}} \frac{\sigma \tau \phi k}{\sigma \tau \phi+1}\right)+\frac{1}{\tau_{d}^{c}}\right] .
$$

Thus we have a method for distinguishing the mechanism responsible for the $1.6 \mathrm{eV}$ emission in silicon-rich silica. By measuring the dependence of luminescence rise time on pump photon flux for a range of samples and plotting reciprocal rise time as a function of $\phi$ it becomes possible to distinguish between the two models.

\section{B. $\mathrm{Er}^{3+}$ emission at $1535 \mathrm{~nm}$}

Turning our attention to the rare-earth ions, the following rate equation governs the population of the ${ }^{4} I_{13 / 2}$ metastable state, and therefore by inference the $1535 \mathrm{~nm}$ emission:

$$
\frac{d N_{\mathrm{RE}}^{*}}{d t}=\left(1-C_{\mathrm{dir}}\right) R^{*}+C_{\mathrm{dir}} \sigma_{\mathrm{Er}} \phi-\frac{N_{\mathrm{RE}}^{*}}{\tau_{d}^{\mathrm{RE}}},
$$

where $N_{\mathrm{RE}}^{*}$ is the concentration of the excited rare-earth ions, $R^{*}$ is the increase of the excited rare-earth population through energy transfer from an exciton to the appropriate excited state (as in Franzò's model, and analogous to $L^{*}$ for the luminescent centers), $C_{\mathrm{dir}}$ is the proportion of excitation of $\mathrm{Er}^{3+}$ attributed to direct absorption of pump photons $\left(0 \leqslant C_{\mathrm{dir}} \leqslant 1\right), \sigma_{\mathrm{Er}}$ is the direct optical absorption cross section of $\mathrm{Er}^{3+}$, and $\tau_{d}^{\mathrm{RE}}$ is the decay lifetime for the $\mathrm{Er}^{3+}$ metastable state, taking into account both radiative and nonradiative processes.

The generation of excitons is governed by Eq. (1), as before, and $R^{*}$ can be written as

$$
R^{*}=\frac{N_{\mathrm{exc}}\left(N_{\mathrm{RE}}-N_{\mathrm{RE}}^{*}\right) \Lambda \eta}{\tau_{\mathrm{tr}}^{\mathrm{RE}}}-\frac{N_{\mathrm{exc}} N_{\mathrm{RE}}^{*} \Lambda}{\tau_{\mathrm{tr}}^{\mathrm{RE}}}(1-\eta),
$$

or

$$
R^{*}=\frac{N_{\mathrm{exc}}\left(\eta N_{\mathrm{RE}}-N_{\mathrm{RE}}^{*}\right) \Lambda}{\tau_{\mathrm{tr}}^{\mathrm{RE}}},
$$

where $\eta(\eta \leqslant 1)$ is a quantum efficiency term which takes into account two factors: first, that the efficiency of the transfer from excitons to rare-earth ions will not be $100 \%$, and second that only a fraction of the excited $\mathrm{Er}^{3+}$ will decay to the appropriate metastable level of $\mathrm{Er}^{3+}$ and hence increase $N_{\mathrm{RE}}^{*}$. The second term in Eq. (12) reflects the probability of upconversion, i.e., the process whereby an erbium ion in the metastable state is excited to higher energy states by transfer from excitons, and therefore does not contribute to the emission process.

We again make the assumption that the population of excitons reaches a steady state well before that of $\mathrm{Er}^{3+}$. Equation (1) can then be solved for the steady state population of excitons which is given by

$$
N_{\mathrm{exc}}=\frac{\sigma \tau \phi k}{\sigma \tau \phi+1} N .
$$

Substituting Eq. (14) into Eq. (13) $R^{*}$ takes the form

$$
R^{*}=\frac{N \Lambda\left(\eta N_{\mathrm{RE}}-N_{\mathrm{RE}}^{*}\right)}{\tau_{\mathrm{tr}}^{\mathrm{RE}}} \frac{\sigma \tau \phi k}{(\sigma \tau \phi+1)} .
$$

Substituting Eq. (15) into Eq. (10), solving the differential equation for a rapidly rising excitation pulse, and applying the boundary conditions $N_{\mathrm{RE}}^{*}(t)$ is given by

$$
\begin{aligned}
N_{\mathrm{RE}}^{*}= & A\left\{1-\exp \left[-\left(\frac{\left(1-C_{\mathrm{dir}}\right) N \Lambda}{\tau_{\mathrm{tr}}^{\mathrm{RE}}} \frac{\sigma \tau \phi k}{\sigma \tau \phi+1}\right.\right.\right. \\
& \left.\left.\left.+C_{\mathrm{dir}} \sigma_{\mathrm{Er}} \phi+\frac{1}{\tau_{d}^{\mathrm{RE}}}\right) t\right]\right\},
\end{aligned}
$$

where 


$$
A=\frac{\left(1-C_{\mathrm{dir}}\right) \frac{N_{\mathrm{RE}} \eta N \Lambda}{\tau_{\mathrm{tr}}^{\mathrm{RE}}} \frac{\sigma \tau \phi k}{(\sigma \tau \phi+1)}+C_{\mathrm{dir}} \sigma_{\mathrm{Er}} \phi N_{\mathrm{RE}}}{\left(1-C_{\mathrm{dir}}\right) \frac{N \Lambda}{\tau_{\mathrm{tr}}^{\mathrm{RE}}} \frac{\sigma \tau \phi k}{(\sigma \tau \phi+1)}+C_{\mathrm{dir}} \sigma_{\mathrm{Er}} \phi+\frac{1}{\tau_{d}^{\mathrm{RE}}}} .
$$

The solution to the erbium rate equation includes a number of parameters that we are unable to measure directly. These include the interaction volume $(\Lambda)$, the transfer time $\left(\tau_{\text {tr }}\right)$, the quantum efficiency of the filling of the erbium metastable state $(\eta)$, and the average number of excitons per silicon nanocrystal $(k)$. However, these can be included in an effective cross section ( $\sigma_{\text {eff }}$ ) for erbium excitation which also takes into account the excitonic absorption cross section $(\sigma)$ and excitonic lifetime $(\tau)$. We choose to define this in the same way as Franzò as

$$
\sigma_{\text {eff }}=\sigma \tau R_{\text {transfer }},
$$

where

$$
R_{\text {transfer }}=\frac{k N \Lambda}{\tau_{\mathrm{tr}}^{\mathrm{RE}}} .
$$

The full solution for the initial growth of $1535 \mathrm{~nm}$ emission with time using Eq. (17) is therefore:

$$
\begin{aligned}
N_{\mathrm{RE}}^{*}= & \frac{\left(1-C_{\mathrm{dir}}\right) N_{\mathrm{RE}} \eta \phi\left(\frac{\sigma_{\mathrm{eff}}}{(\sigma \tau \phi+1)}\right)+C_{\mathrm{dir}} \sigma_{\mathrm{Er}} \phi N_{\mathrm{RE}}}{\left(1-C_{\mathrm{dir}}\right) \phi\left(\frac{\sigma_{\mathrm{eff}}}{(\sigma \tau \phi+1)}\right)+C_{\mathrm{dir}} \sigma_{\mathrm{Er}} \phi+\frac{1}{\tau_{d}^{\mathrm{RE}}}} \\
& \times\left(1-\exp \left\{-\left[\left(1-C_{\mathrm{dir}}\right) \phi\left(\frac{\sigma_{\mathrm{eff}}}{(\sigma \tau \phi+1)}\right)\right.\right.\right. \\
& \left.\left.\left.+C_{\mathrm{dir}} \sigma_{\mathrm{Er}} \phi+\frac{1}{\tau_{d}^{\mathrm{RE}}}\right] t\right\}\right) .
\end{aligned}
$$

Equation (20) describes the growth of the excited erbium population with time and thus, the photoluminescence intensity at $1535 \mathrm{~nm}$. Using the above model it is possible to study both $\mathrm{Er}^{3+}$-doped stoichiometric silica samples in which erbium is excited only directly by the absorption of pump photons $\left(C_{\mathrm{dir}}=1\right)$ as well as $\mathrm{Er}^{3+}$-doped silicon nanoclusters which exhibit indirect excitation of erbium $\left(C_{\mathrm{dir}}\right.$ $<1)$. In Eq. (20) the exponential term describes the rise time of the erbium emission as a function of $\phi$, whereas the preexponential term describes the photoluminescence intensity as a function of $\phi$.

\section{RESULTS AND DISCUSSION}

\section{A. $1.6 \mathrm{eV}$ emission}

Figure 2 shows the dependence of the rise time of the $1.6 \mathrm{eV}(724 \mathrm{~nm})$ emission on $488 \mathrm{~nm}$ pump photon flux for implanted films containing 5 and 15 at. \% excess silicon (no erbium) and 15 at. \% excess silicon with 0.5 at. \% erbium. In all cases, the plots are straight lines within the experimental error. Although this is a relatively small sample and more experiments are necessary to be conclusive, the results are indicative of a first-order process, as described by Eq. (3):

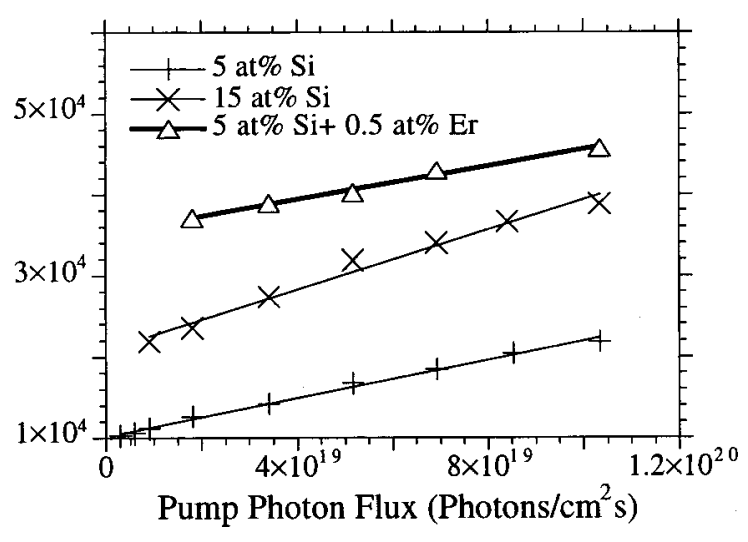

FIG. 2. Plots of reciprocal rise times of $1.6 \mathrm{eV}$ emission as a function of excitation photon flux for implanted samples with and without erbium. Pump wavelength $=488 \mathrm{~nm}$.

i.e., the absorbing species is that which emits at $1.6 \mathrm{eV}$. This supports the contention that the source of the $1.6 \mathrm{eV}$ emission is radiative recombination of excitons confined within the silicon nanoclusters. Of course, it is also possible that the absorption cross section may be very small, leading to a very slight degree of curvature which may be mistaken for a straight line. However, this result should be considered in conjunction with two other observations: the redshift of luminescence peak energy with annealing, ${ }^{5}$ and the dependence of luminescence lifetime on the presence of rare-earth ions. If we assume that the $1.6 \mathrm{eV}$ emission is due to radiative recombination of excitons, which are the same species that couple to the optically active erbium ions, then clearly these two processes are in competition. An increase in erbium concentration will therefore reduce the luminescence lifetime of the excitonic emission. If, however, the $1.6 \mathrm{eV}$ emission arises from luminescent centers not coupled to the rare-earth ions, erbium concentration and $1.6 \mathrm{eV}$ luminescent lifetime will be independent. We have studied the effect of implanting erbium into silicon-rich silica on the lifetime of the $1.6 \mathrm{eV}$ emission: our results for the 5 at. \% excess silicon sample show a clear decrease in luminescence lifetime from $98 \mu$ s without erbium to $28 \mu \mathrm{s}$ in the presence of 0.5 at. $\%$ erbium. Note that the erbium-doped sample was annealed at $900{ }^{\circ} \mathrm{C}$ following rare-earth implantation to remove implantation related damage. While we do not have data for a range of erbium concentrations, this result is indicative of strong coupling between the $1.6 \mathrm{eV}$ emitter and the rare-earth ion. We therefore tentatively conclude that the $1.6 \mathrm{eV}$ emission is due to radiative recombination of confined excitons. Franzò et al. ${ }^{10}$ have made similar measurements and have detected no dependence of excitonic lifetime on erbium concentration. They therefore conclude that the species responsible for this emission is not that which couples to the erbium ion. It is possible that the disagreement between our results and those of Franzo et al. may be due to the much lower erbium fluxes employed in their work. They report lifetime data for maximum erbium fluxes of $1 \times 10^{13} \mathrm{~cm}^{-2}$ at $300 \mathrm{keV}$ compared to our implants at up to $2.4 \times 10^{15} \mathrm{~cm}^{-2}$ and $380 \mathrm{keV}$. Thus, while not conclusive, the available evidence supports the excitonic recombination model. 
Looking in detail at the data for the implanted sample containing 5 at. $\%$ excess silicon and 0.5 at. \% erbium, a straight-line fit gives an excitonic absorption cross section of $1.02 \times 10^{-16} \mathrm{~cm}^{2}$ and an excitonic lifetime of $28.3 \mu \mathrm{s}$. This produces a value of the combined parameter $\sigma \tau$ of 2.89 $\times 10^{-21} \mathrm{~cm}^{2} \mathrm{~s}$. The excitonic generation process in silicon nanoclusters has recently been studied and the cross section determined ${ }^{33,34}$ For a pump wavelength in the region of 476 $\mathrm{nm}$ and emission around $1.6 \mathrm{eV}$, the excitonic absorption cross section has been measured by Kovalev et al. to be around $2 \times 10^{-16} \mathrm{~cm}^{2}$. Priolo's group quotes a figure for isolated silicon nanoclusters of $1.8 \times 10^{-16} \mathrm{~cm}^{2}$ for a pump wavelength of $488 \mathrm{~nm}$. In the same study, it was found that the presence of erbium increases this cross section by a factor of 4 . These figures are in very good agreement with the values determined in our study, though it should be noted that values of $\sigma$ quoted Kovalev's study are extremely sensitive to both excitation and emission wavelengths. Fitting the $1.6 \mathrm{eV}$ intensity data for this sample with the preexponential term from Eq. (3) (i.e., the steady-state population of excitons) yields an upper limit on the value of the combined parameter $\sigma \tau$ of $1 \times 10^{-21} \mathrm{~cm}^{2} \mathrm{~s}$. Given the errors associated with making these measurements, we are confident that the two data sets are consistent, and are encouraged by their agreement with data from Refs. 33 and 34 .

\section{B. $1.5 \mu \mathrm{m}$ emission}

Using as inputs to our model four data sets for each sample: (i) $1.6 \mathrm{eV}$ reciprocal rise time as a function of photon flux, (ii) $1.6 \mathrm{eV}$ intensity as a function of photon flux, (iii) $1535 \mathrm{~nm}$ reciprocal rise time as a function of photon flux, and (iv) $1535 \mathrm{~nm}$ intensity as a function of photon flux, we can in principle obtain by iteration values of $\sigma \tau$ and $\sigma_{\text {eff }}$ that should be consistent. In practice, limitations on luminescence signal or the time resolution of our system mean that not all samples provide all four data sets. However, as far as is possible, we have obtained these data for both PECVD and implanted samples.

\section{Evaluation of effective erbium absorption cross section: PECVD material}

We have obtained data sets (ii), (iii), and (iv) for a sample produced by PECVD and annealed at $1100^{\circ} \mathrm{C}$. Reciprocal rise time data for a pump wavelength of $476 \mathrm{~nm}$ are shown in Fig. 3, along with a fit to the data from the exponential term of Eq. (20). The intensity data and fits using the preexponential term of Eq. (20) are shown in Fig. 4 for pump wavelengths of 476 and $488 \mathrm{~nm}$ (i.e., predominantly indirect and predominantly direct excitation, respectively).

Looking first at the $1.6 \mathrm{eV}$ intensity as a function of photon flux [data set (ii)], this is very close to a straight line over the range of photon fluxes studied. This being the case, it is not possible to obtain an accurate value for $\sigma \tau$ over this range of photon fluxes, though an upper limit can be deduced. Fitting the data in Fig. 4 using Eq. (3) gives upper limits on $\sigma \tau$ of $5.6 \times 10^{-21}$ and $1.9 \times 10^{-21} \mathrm{~cm}^{2} \mathrm{~s}$ for pump wavelengths of 488 and $476 \mathrm{~nm}$, respectively: using these in equation (20) to fit the data in Figs. 3 and 4 yields the values

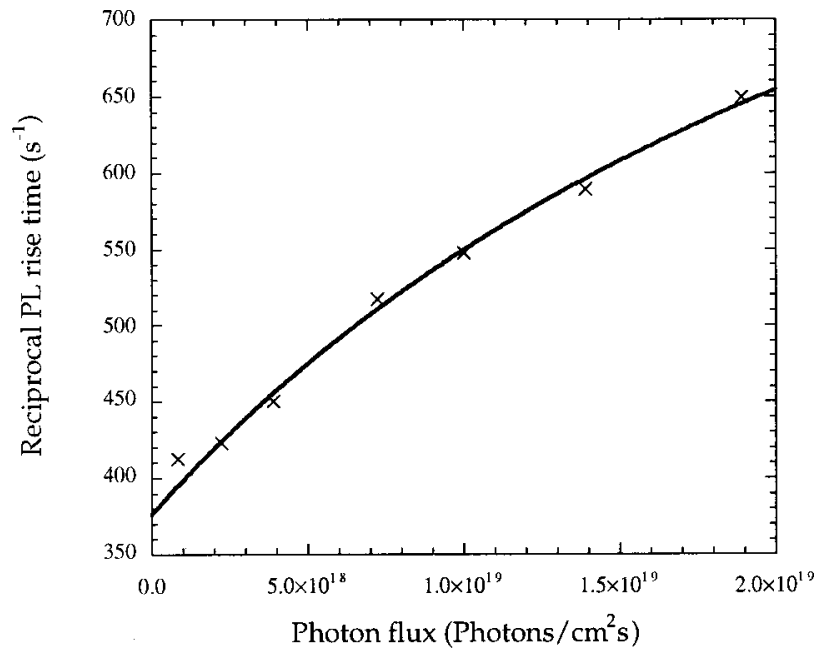

FIG. 3. Plot of reciprocal rise time of $1535 \mathrm{~nm}$ emission from PECVD produced sample containing 7 at. $\%$ excess silicon and 1 at. $\%$ erbium. Pump wavelength $=476 \mathrm{~nm}$. Crosses: experimental data; solid line: fit using Eq. (20).

of $\sigma_{\text {eff }}$ given in Table I. The results are consistent and give reasonable agreement. We are therefore confident that this model gives a reliable measure of the effective cross section for indirect excitation of erbium. We are further encouraged by the agreement between our values for $\sigma_{\text {eff }}$ and those published recently by Priolo's group $\left(1.1 \times 10^{-16} \mathrm{~cm}^{2}\right) .{ }^{34}$
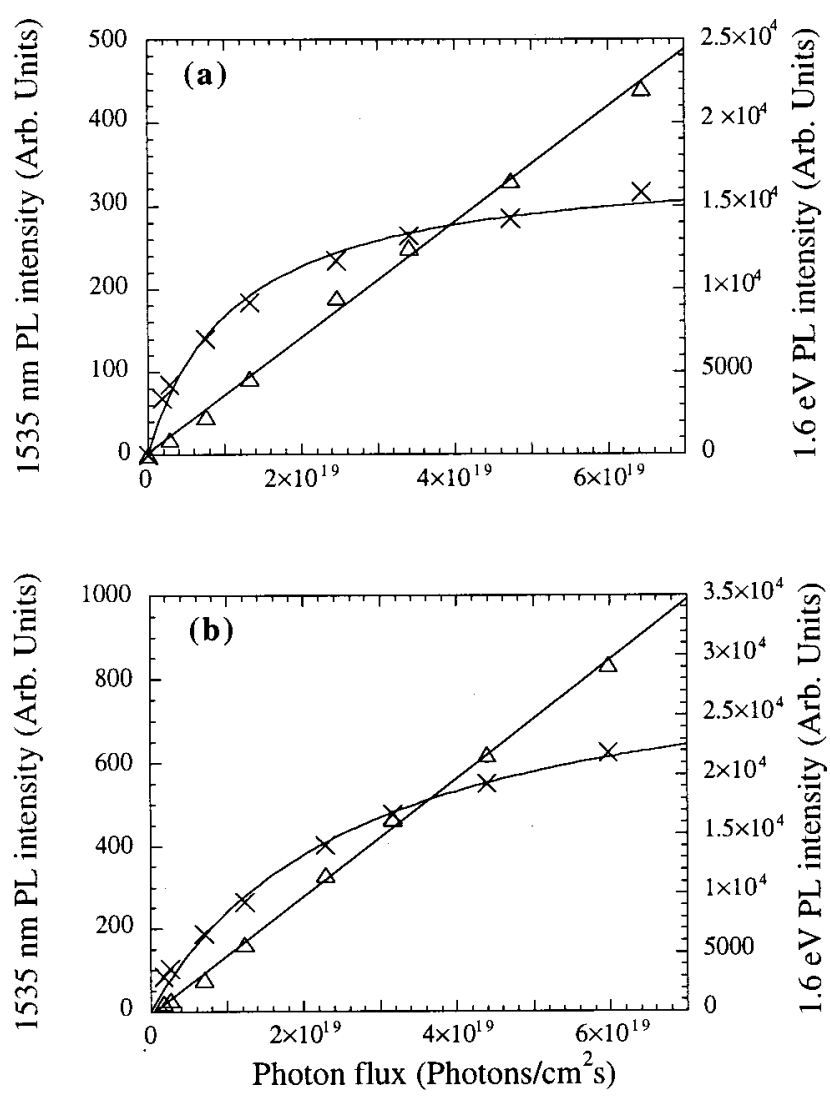

FIG. 4. Dependence of emission at $1.6 \mathrm{eV}$ (724 nm: (triangles) and $1535 \mathrm{~nm}$ (crosses) on pump photon flux for a PECVD sample containing 7 at. $\%$ excess silicon and 1 at. \% erbium. Pump wavelength $=476 \mathrm{~nm}$ (a) and 488 nm (b). 
TABLE I. Effective Er absorption cross sections determined from fits of experimental data using Eq. (20) for the PECVD 7 at. \% Si, 1 at. \% $\mathrm{Er}$ sample.

\begin{tabular}{lccc}
\hline \hline $\begin{array}{l}\text { Measurement } \\
\text { (vs. photon flux) }\end{array}$ & $\begin{array}{c}\text { Pump } \\
\text { wavelength } \\
(\mathrm{nm})\end{array}$ & $\sigma \tau$ (exciton) & $\begin{array}{c}\text { Effective erbium } \\
\text { cross section }\end{array}$ \\
\hline $1.6 \mathrm{eV}$ intensity & 476 & $<1.9 \times 10^{-21} \mathrm{~cm}^{2} \mathrm{~s}$ \\
$1535 \mathrm{~nm}$ rise time & 476 & as above & $1.67 \times 10^{-17} \mathrm{~cm}^{2}$ \\
$1535 \mathrm{~nm}$ intensity & 476 & as above & $2.45 \times 10^{-17} \mathrm{~cm}^{2}$ \\
$1.6 \mathrm{eV}$ intensity & 488 & $<5.6 \times 10^{-21} \mathrm{~cm}^{2} \mathrm{~s}$ & \\
$1535 \mathrm{~nm}$ rise time & 488 & as above & $5.30 \times 10^{-17} \mathrm{~cm}^{2}$ \\
$1535 \mathrm{~nm}$ intensity & 488 & as above & $7.33 \times 10^{-17} \mathrm{~cm}^{2}$ \\
\hline \hline
\end{tabular}

Note that the values of $C_{\text {dir }}$ were obtained from the photoluminescence excitation spectrum of this sample, ${ }^{25}$ and were measured to be 0.75 and 0.16 at pump wavelengths of 488 and $476 \mathrm{~nm}$, respectively. $\tau_{d}^{\mathrm{RE}}$ was measured to be $2.5 \mathrm{~ms}$, and the cross sections for direct optical absorption by erbium were taken to be $1 \times 10^{-21}$ and $8 \times 10^{-21} \mathrm{~cm}^{2}$ at 476 and $488 \mathrm{~nm}$, respectively.

\section{Evaluation of effective erbium absorption cross section: Implanted material}

Due to small signals, it was not possible to measure the reciprocal rise times of the $1535 \mathrm{~nm}$ emission for the three implanted samples. However, having validated the theory by obtaining consistent values of $\sigma_{\text {eff }}$ for the PECVD sample, we fitted the $1.6 \mathrm{eV}$ intensity data using Eq. (3) and the $1535 \mathrm{~nm}$ intensity versus pump photon flux data using Eq. (20). The results are presented in Table II. Of course, it should be emphasized that the values for $\sigma_{\text {eff }}$ quoted have been obtained for data sets (i) and (iv) only, and have not been cross checked with measurements of reciprocal rise time of $1535 \mathrm{~nm}$ emission. However, they are indicative and show an enhancement of the $\mathrm{Er}^{3+}$ absorption cross section of approximately three orders of magnitude compared to stoichiometric silica samples.

A comparison of the saturation behavior of the $1535 \mathrm{~nm}$ emission in stoichiometric and silicon-rich samples suggests that in the latter the excitation of $\mathrm{Er}^{3+}$ ions takes place predominantly through energy transfer from $n c$-Si. The exchange of excitation to $\mathrm{Er}^{3+}$ depends on the $\mathrm{Er}^{3+}$ concentration, the effective absorption cross section, and the separation between nanoclusters and erbium ions. Our observation of a large cross section for the combined exciton generation/excitation exchange process suggests that satura-

TABLE II. Effective Er absorption cross section as a function of excess silicon content for samples produced by ion implantation.

Excess silicon content Pump wavelength Effective erbium cross section (at. \%) $(\mathrm{nm})$ $\left(\mathrm{cm}^{2}\right)$

\begin{tabular}{rll}
\hline 5 & 476 & $1.40 \times 10^{-18}$ \\
10 & 476 & $4.40 \times 10^{-18}$ \\
15 & 476 & $6.41 \times 10^{-18}$ \\
5 & 488 & $1.01 \times 10^{-18}$ \\
10 & 488 & $2.19 \times 10^{-18}$ \\
15 & 488 & $5.70 \times 10^{-18}$ \\
\hline
\end{tabular}
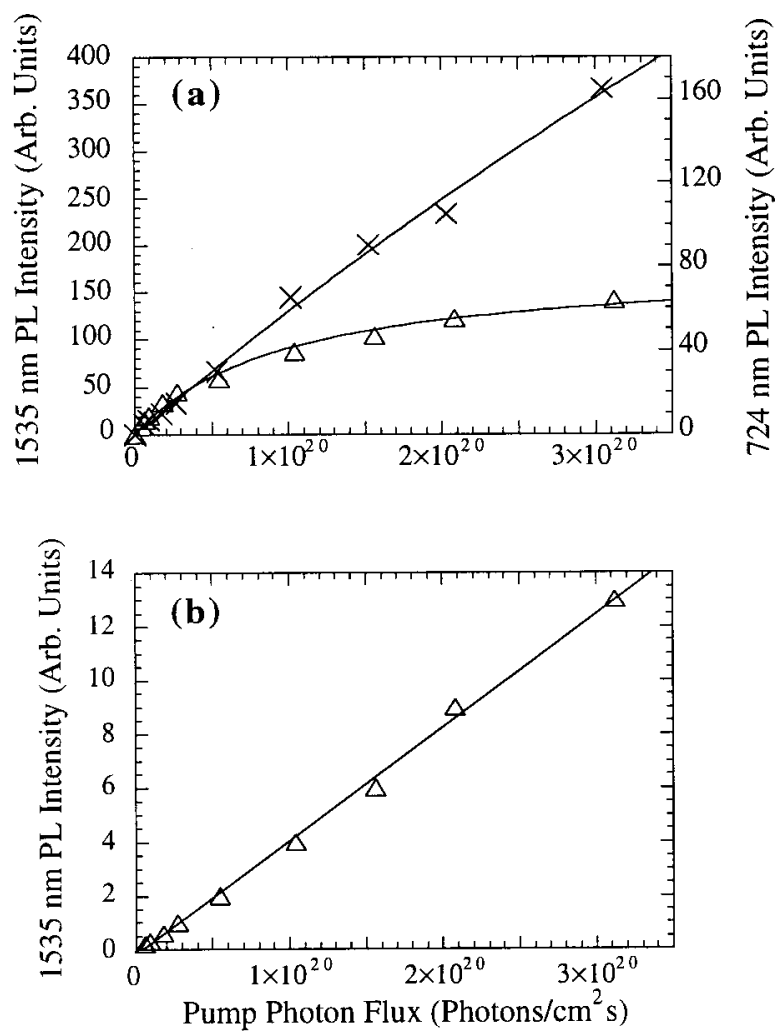

FIG. 5. (a) Dependence of emission at $1.6 \mathrm{eV}$ ( $724 \mathrm{~nm}$ : (crosses) and 1535 $\mathrm{nm}$ (triangles) on $476 \mathrm{~nm}$ pump photon flux for an implanted sample containing 10 at. \% excess silicon and 0.5 at. \% erbium; (b) similar plot for $1535 \mathrm{~nm}$ emission for a stoichiometric silica sample implanted with 0.5 at. $\%$ erbium.

tion of the $1535 \mathrm{~nm}$ emission is due to the fact that the luminescence is limited by the availability of $\mathrm{Er}^{3+}$ in close proximity to silicon nanocrystals. On the other hand, the luminescence intensity at $1535 \mathrm{~nm}$ does not saturate for the stoichiometric $\mathrm{SiO}_{2}$ sample implanted with the same erbium concentration (0.5 at. \%) (Fig. 5). This is because the cross section for direct excitation of $\mathrm{Er}^{3+}$ ions is much smaller, and therefore the luminescence yield is not constrained by the availability of $\mathrm{Er}^{3+}$ ions but rather by the small absorption cross section. Consequently, luminescence saturation is only seen at much higher photon fluxes. This also explains the small photoluminescence yield exhibited by the stoichiometric $\mathrm{SiO}_{2}$ sample.

The values we have obtained for $\sigma_{\text {eff }}$ for silicon-rich samples are up to four orders of magnitude larger than the literature value for the $\mathrm{Er}^{3+}$ cross section in silica for direct optical absorption at either 488 or $980 \mathrm{~nm}$. Indeed, our values are approaching the $\mathrm{Er}^{3+}$ optical absorption cross section in bulk silicon $\left(1 \times 10^{-15} \mathrm{~cm}^{2}\right)$. However, the luminescence yield in bulk silicon is much lower than in either silica or silicon-rich silica due to the lack of $\mathrm{Er}^{3+}$ coordination with oxygen and the strong nonradiative decay channels present in silicon.

The large effective cross section measured in this work explains why the presence of silicon nanoclusters in silica enhances $\mathrm{Er}^{3+}$ photoluminescence even at wavelengths that correspond to direct optical transitions. Even at these wavelengths the indirect channel is strong and dominates for excess silicon contents greater than around 10 at. \%. This is an 
important point for the eventual technological exploitation of this material.

Our results indicate an increase in effective cross section as the amount of excess silicon increases. This is consistent with the hypothesis that, at high excess silicon concentrations, $\mathrm{Er}^{3+}$ ions are incorporated within the silicon nanoclusters. Evidence in the form of reduced luminescence lifetimes and lower photoluminescence yields supports this. As the $\mathrm{Er}^{3+}$ ions experience a more silicon-rich environment, the luminescence lifetime, effective cross section and luminescence yield begin to approach the values seen in bulk silicon.

\section{CONCLUSION}

We have presented a model for the excitation coupling between silicon nanoclusters and $\mathrm{Er}^{3+}$ ions that allows us to obtain an effective cross section for the indirect excitation of the $\mathrm{Er}^{3+}$ metastable state. Because we do not have access to information about which of the erbium upper energy levels are coupled to the silicon nanoclusters, this must remain an effective cross section. However, it is a useful figure-of-merit to characterize the exchange process and allow comparison with direct optical excitation. For our silicon-rich samples, $\sigma_{\text {eff }}$ is up to four orders of magnitude higher than the $\mathrm{Er}^{3+}$ direct optical absorption cross-section in silica at $488 \mathrm{~nm}$. These figures agree well with data published elsewhere in the literature, ${ }^{10,34}$ although our model is different from that used by other groups in that it explicitly limits the number of excitons that can exist on a single silicon nanocluster. Significantly, our model is generic in that it describes the interaction between any semiconductor nanocluster and coupled emitter: it is not specific to the $n c-\mathrm{Si}_{\mathrm{Er}}{ }^{3+}$ system, and it takes into account both direct and indirect excitation.

We have also used the model to draw some conclusions about the nature of the species responsible for the $1.6 \mathrm{eV}$ emission band associated with silicon nanoclusters. Within the resolution of our measurements, and in agreement with our previous work, our conclusion is that this is produced by the radiative recombination of confined excitons. The cross sections for optical absorption by silicon nanoclusters determined in this study are in good agreement with those produced by other workers. 33,34

\section{ACKNOWLEDGMENTS}

The authors are grateful for the funding provided for this work by the Engineering and Physical Sciences Research Council.
${ }^{1}$ A. Polman, J. Appl. Phys. 82, 1 (1997).

${ }^{2}$ L. T. Canham, Appl. Phys. Lett. 57, 1046 (1990).

${ }^{3}$ T. Shimizu-Iwayama, K. Fujita, S. Nakao, K. Saitoh, T. Fujita, and N. Itoh, J. Appl. Phys. 75, 7779 (1994).

${ }^{4}$ J. G. Zhu, C. W. White, J. D. Budai, S. P. Withrow, and Y. Chen, J. Appl. Phys. 78, 4386 (1995).

${ }^{5}$ A. J. Kenyon, P. F. Trwoga, C. W. Pitt, and G. Rehm, J. Appl. Phys. 79, 9291 (1996)

${ }^{6}$ K. S. Min, K. V. Shcheglov, C. M. Yang, H. A. Atwater, M. L. Brongersma, and A. Polman, Appl. Phys. Lett. 69, 2033 (1996).

${ }^{7}$ F. Iaconia, G. Franzò, and C. Spinella, J. Appl. Phys. 87, 1295 (2000).

${ }^{8}$ S. Guha, J. Appl. Phys. 84, 5210 (1998).

${ }^{9}$ M. Y. Valakh, V. A. Yukhimchuk, V. Y. Bratus, A. A. Konchits, P. L. F. Hemment, and T. Komoda, J. Appl. Phys. 85, 168 (1999).

${ }^{10}$ G. Franzò, V. Vinciguerra, and F. Priolo, Appl. Phys. A: Mater. Sci. Process. 69, 3 (1999).

${ }^{11}$ T. Shimizu-Iwayama, N. Kurumado, D. E. Hole, and P. D. Townsend, J. Appl. Phys. 83, 6018 (1998).

${ }^{12}$ Y. Kanemitsu, Thin Solid Films 276, 44 (1996).

${ }^{13}$ V. V. Afanas'ev and A. Stesmans, Phys. Rev. B 59, 2025 (1999).

${ }^{14}$ R. T. Collins, P. M. Fauchet, and M. A. Tischler, Phys. Today 50, 24 (1997).

${ }^{15}$ P. F. Trwoga, A. J. Kenyon, and C. W. Pitt, Electron. Lett. 32, 1703 (1996).

${ }^{16}$ G. S. Tompa, D. C. Morton, B. S. Sywe, Y. Lu, E. W. Forsythe, J. A. Ott, D. Smith, J. Khurgin, and B. A. Khan, Mater. Res. Soc. Symp. Proc. 358, 701 (1995).

${ }^{17}$ G. G. Qin, A. P. Li, B. R. Zhang, and B.-Chen Li, J. Appl. Phys. 78, 2006 (1995).

${ }^{18}$ A. J. Kenyon, P. F. Trwoga, M. Federighi, and C. W. Pitt, J. Phys.: Condens. Matter 6, L319 (1994).

${ }^{19} \mathrm{X}$. Wu, U. Hommerich, F. Namavar, and A. M. Cremins Costa, Appl. Phys. Lett. 69, 1903 (1996).

${ }^{20}$ M. Fujii, M. Yoshida, Y. Kanzawa, S. Hayashi, and K. Yamamoto, Appl. Phys. Lett. 71, 1198 (1997).

${ }^{21}$ J. H. Shin, M. Kim, S. Seo, and C. Lee, Appl. Phys. Lett. 72, 1092 (1998).

${ }^{22}$ L. Tsybeskov, S. P. Duttagupta, K. D. Hirschman, P. M. Fauchet, K. L. Moore, and D. G. Hall, Appl. Phys. Lett. 70, 1790 (1997).

${ }^{23}$ M. Fujii, M. Yoshida, S. Hayashi, and K. Yamamoto, J. Appl. Phys. 84, 4525 (1998).

${ }^{24}$ J. H. Shin, S. Seo, S. Kim, and S. G. Bishop, Appl. Phys. Lett. 76, 1999 (2000).

${ }^{25}$ C. E. Chryssou, A. J. Kenyon, T. S. Iwayama, C. W. Pitt, and D. E. Hole, Appl. Phys. Lett. 75, 2011 (1999).

${ }^{26}$ G. Franzò, D. Pacifici, V. Vinciguerra, F. Priolo, and F. Iaconia, Appl. Phys. Lett. 76, 2167 (2000).

${ }^{27}$ P. G. Kik and A. Polman, J. Appl. Phys. 88, 1992 (2000).

${ }^{28}$ A. J. Kenyon, C. E. Chryssou, and C. W. Pitt, Appl. Phys. Lett. 76, 688 (2000).

${ }^{29}$ G. Qin, G. G. Qin, and S. H. Wang, J. Appl. Phys. 85, 6738 (1999).

${ }^{30}$ A. J. Kenyon, C. E. Chryssou, and C. W. Pitt, Proc. SPIE 4282, 185 (2001).

${ }^{31}$ N. Sharma, V. J. Keast, T. S. Iwayama, I. Boyd, and C. J. Humphreys, Inst. Phys. Conf. Ser. 161, 589 (1999).

${ }^{32}$ S. K. Bera, S. Chaudhuri, A. K. Bandyopadhyay, B. R. Chakraborty, and A. K. Pal, J. Phys. D 34, 273 (2001).

${ }^{33}$ D. Kovalev, J. Diener, H. Heckler, G. Polisski, N. Künzner, and F. Koch, Phys. Rev. B 61, 4485 (2000).

${ }^{34}$ F. Priolo, G. Franzo, D. Pacifici, V. Vinciguerra, F. Iacona, and A. Irrera, J. Appl. Phys. 89, 264 (2000). 